\title{
Circuit
}

Musiques contemporaines

\section{ECM+Génération 2016 : quatre compositeurs, quatre sons}

\section{Pierre-Luc Senécal}

Volume 27, numéro 1, 2017

URI : https://id.erudit.org/iderudit/1039675ar

DOI : https://doi.org/10.7202/1039675ar

Aller au sommaire du numéro

Éditeur(s)

Les Presses de l’Université de Montréal

ISSN

1183-1693 (imprimé)

1488-9692 (numérique)

Découvrir la revue

Citer ce document

Senécal, P.-L. (2017). ECM+ Génération 2016 : quatre compositeurs, quatre sons. Circuit, 27(1), 77-80. https://doi.org/10.7202/1039675ar

Ce document est protégé par la loi sur le droit d'auteur. L'utilisation des services d’Érudit (y compris la reproduction) est assujettie à sa politique d'utilisation que vous pouvez consulter en ligne.

https://apropos.erudit.org/fr/usagers/politique-dutilisation/ 


\title{
ECM+ Génération 2016 : quatre compositeurs, quatre sons
}

\author{
Pierre-Luc Senécal
}

\section{Génération : un projet pérenne de I'ЕСм+}

Après 22 ans d'existence, le concours bisannuel Génération ${ }^{1}$ de l'Ensemble contemporain de Montréal (ECM+) poursuit l'aventure de l'accompagnement et de la promotion de la jeune création musicale canadienne. Pour cette quatorzième édition, le jury du concours, composé de producteurs et de compositeurs provenant de tout le pays, a sélectionné parmi une cinquantaine de candidatures les compositeurs Symon Henry, Adam Scime et Taylor Brook, et la compositrice Sabrina Schroeder. Tous dotés d'une solide formation et d'une démarche affirmée, ils avaient à leur disposition un ensemble de dix musiciens dirigés par la pétillante Véronique Lacroix et près d'un an pour composer une pièce. Chacun à sa façon, ils sont arrivés au bout d'un projet de composition échafaudé en deux temps: des ateliers publics et une tournée canadienne ${ }^{2}$.

\section{Les ateliers : dans la "boîte noire" de la création}

Loriginalité de Génération provient de sa formule. Alors que de nombreux concours et ateliers de composition sélectionnent les compositeurs en fonction de pièces déjà écrites ou à écrire au cours d'une seule résidence ${ }^{3}$ Génération débute avec une période de travail en amont. Les compositeurs rencontrent les musiciens de l'ensemble lors d'une présentation publique afin de se renseigner sur des techniques étendues spécifiques aux instruments. Les compositeurs font donc leurs découvertes en même temps que les auditeurs. Il s'agit d'une occasion rare pour le public, car il peut découvrir les dessous d'un moment fondamental de la création musicale: le travail avec les interprètes.

Si les compositeurs cherchent à éclaircir comment produire tel ou tel effet sur un instrument et comment le noter, c'est qu'ils sont en "quête de son », une démarche proche de la pratique en musique électroacoustique $^{4}$. Il n'est donc pas surprenant que cette édition 2016 de Génération ait été dirigée, dès les ateliers, vers un travail en profondeur sur le sonore. En effet, les quatre compositeurs avaient quatre façons différentes d'explorer, par la musique instrumentale, les propriétés du son. C'est sous cet angle qu’a été rédigé ce compte rendu.

\section{Tournée Génération 2016 : l'ECM + en concert à Montréal}

Après avoir sillonné le Canada, l'ECM+ a finalement rejoint Montréal. Commençant sa tournée à Banff, en Colombie-Britannique, où il venait de terminer une résidence de cinq jours, l'ensemble a pris la route vers Edmonton, puis Victoria et Vancouver avant de revenir 
au bercail. Avec encore Toronto, London, Ottawa et Québec à parcourir, l'ensemble réalisait donc un concert «mi-temps» dans la salle du Conservatoire, bien remplie par les membres de la communauté locale de la musique contemporaine.

La soirée était animée par le compositeur, performeur et improvisateur vocal Gabriel Dharmoo. Entre chacune des pièces, il invitait les compositeurs à parler brièvement de leur composition. S'il s'adressait au public de façon plus informelle, les compositeurs donnaient des réponses préparées et indiquées sur des feuilles qu'ils pouvaient lire d'un coup d'œil discret. Notons l'effort de faire parler en français les compositeurs, Symon Henry étant le seul ayant le français pour langue natale.

\section{Symon Henry: adepte de la partition graphique}

Symon Henry ${ }^{5}$ maintient que le dessin est la meilleure façon de communiquer ses intentions à l'interprète. Il utilise le fusain, le pastel et le graphite pour «noter le son» et tracer, sur ses partitions, les transformations très «organiques» qu'il souhaite entendre. Il se fait alors artiste visuel, et adopte une démarche qui le rapproche de l'ingénieur sonore. En manipulant différents effets audionumériques et microphones, l'ingénieur pose des gestes qui ont une incidence directe sur l'«argile sonore» qu'il sculpte par filtrage, compression, mixage, etc. En traçant des «lignes musicales» sur de grandes feuilles accrochées aux murs de son appartement, Henry établit avec la musique un rapport plutôt haptique, quasi ludique.

Le compositeur Georges Bœuf expliquait qu'en utilisant la bande magnétique, les ciseaux et les collants, il touchait presque à la matière du son ${ }^{6}$. Michel Chion disait même que «si les sons tachaient, on verrait les compositeurs sortir barbouillés des studios, comme les peintres ${ }^{7} »$. Est-ce si farfelu d'imaginer Henry dans son atelier, modelant le son de ses mains tachées de graphite?
Dans sa pièce, Debout, un respir grand comme, la matière dense et riche que les musiciens s'échangent a quelque chose de profondément gestuel. Lignes, textures planantes, chants qui émergent du silence... Le son est fébrile, pétulant. Remarquablement bien structurée, la musique «respire » et on peine à croire qu'un dessin en est l'origine. La justesse de la démarche de Henry devient apparente dans ses solos de violon. Rappelant des airs chantés, ils sont d'une étonnante organicité et exigeraient pour leur interprétation des indications nettement plus difficiles à communiquer s'ils étaient notés de façon conventionnelle.

\section{Adam Scime : architecture et espaces}

La musique de Scime ${ }^{8}$ (prononcé "Chimé») paraîtra également familière à bien des compositeurs acousmatiques. Lorsqu'il parle de son travail, Scime utilise des mots comme «portes», «escaliers », «entrées» et «création d'espaces imaginaires». Ces termes désignent l'une de ces techniques d'écriture caractéristiques de la musique électroacoustique : le montage. Grâce à celui-ci, les électroacousticiens peuvent, en quelques clics dans le séquenceur, faire apparaître et disparaître des objets sonores et des espaces, par exemple. Les sons qu'ils assemblent peuvent plonger les auditeurs dans des lieux inouïs, et le compositeur joue alors à un jeu de «portes vers des "ailleurs" » qu'il ouvre ou claque à volonté. En ce sens, ces compositeurs sont des prestidigitateurs.

La musique de Scime rappelle également la musique électroacoustique, car elle est un exemple de haut niveau d'articulation de l'espace. En concert, la musique est une architecture dont on perçoit clairement les différentes strates. Véritable polyphonie de techniques avancées, les sons s'empilent comme des briques, donnant la musique la plus articulée et énergique de la soirée. Même dans les moments d'accalmie, la tension subsiste. Séquences-jeu, questionréponse, orchestration par timbres complémentaires 
superposés... Cette façon de subdiviser la musique et de la distribuer à chacun des interprètes placés sur scène n'est pas étrangère à l'acousmonium sur lequel François Bayle diffuse sa musique ${ }^{9}$. Dans ce dispositif, des haut-parleurs de toute sorte (haut-parleurs de voiture, enceintes $h i-f i$, etc.) sont placés dans l'espace de concert, et colorent chacun à leur façon les différentes régions du spectre d'une pièce.

\section{Schroeder: être dans le son}

L'acousmonium permet l'expérience de l'immersion sonore, une sensation d'enveloppement maintenant associée aux pièces acousmatiques diffusées sur orchestre de haut-parleurs. Cette propriété enveloppante des sons est au cœur de la pièce de Sabrina Schroeder ${ }^{10}$. Lauréate du Prix du public Génération 2016, c'est sans conteste celle qui s'associait le plus fortement à la pratique de l'art sonore de par son intérêt pour l'intériorité du son, le grain et la spatialisation. En somme, des termes avec lesquels les disciples de Pierre Schaeffer sont familiers! En demandant aux interprètes de jouer les cordes complètement détendues de leur instrument, Schroeder affirmait à la blague, lors de son entretien avec Gabriel Dharmoo, pouvoir déployer les «super-pouvoirs» d'une scordatura extrême, explorant ainsi le «ressenti viscéral des sons ».

Dans Bone Games - Shy Garden, la compositrice met en action un système de transducteurs. Leur vibration «active» de grandes boîtes vides placées sur scène, soutenant la musique d'un grondement sourd. Très usité pour combler le registre grave dans les pièces électroacoustiques, ce genre d'«objet sonore » se remarque particulièrement, car les habitués de la musique strictement instrumentale savent qu'aucun instrument, hormis une pédale d'orgue, ne peut soutenir aussi longuement une note aussi grave.

D'emblée, l'introduction de l'œuvre se démarque. Le souffle des archets rappelle des passages typiques du répertoire électroacoustique. On est pris de surprise lorsque le grondement des boîtes change de hauteur. Le système marche visiblement mieux que lors de l'atelier de février 2016, où le dispositif produisait des basses envahissantes, hors de contrôle. À ppp de dynamique, un surplus de trois décibels paraîtrait de trop. Même si la concision et l'économie de moyens de la pièce passent de vertus à longueurs vers la fin, la plage dynamique demeurant assez similaire, la pièce maintient les auditeurs dans une sorte de douce tension.

\section{Taylor Brook : héros romantique}

Lauréat du Prix du jury Génération 2016, Taylor Brook ${ }^{11}$ s'inscrit dans une conception plus conventionnelle de la musique instrumentale. En entrevue, il va jusqu'à affirmer: «Je ne suis pas un compositeur "sonore". Je ne m'intéresse pas au son de la même façon qu'un compositeur acousmatique.» Effectivement, sa musique puise plutôt dans l'exotisme. Friand de microtons, dont il a approfondi sa connaissance lors d'un voyage en Inde, Brook s'inspire aussi des cultures de Chine et d'Afrique centrale. Pour lui, il ne s'agit pas de s'approprier une culture en faisant un pastiche, mais plutôt de combiner différentes traditions musicales afin de créer une tradition imaginée.

La pièce Tirant Lo Blanc témoigne de l'intérêt du compositeur pour la figure héroïque du chevalier dans les contes. Cette figure s'apparente à celle du soliste, une sorte de protagoniste musical qui échappe même aux directives du chef. Ici, c'est la flûtiste MarieHélène Brault qui jouait le rôle de l'héroïne. Mélange de doigtés microtonaux et de sonorités debussystes, la pièce nécessite une scordatura précise au cent ${ }^{12}$ près, ceci afin de mettre en valeur l'harmonie de la pièce et d'alterner entre intervalles justes et naturels. À la fois familière de par son caractère impressionniste et étrangère en raison des dissonances microtonales, on croit assister à l'épanouissement d'une fleur aux couleurs inusitées. Cette harmonie luxuriante se faufile sous 
la masse, se poursuit dans les crescendos très denses, et donne à la musique comme une teinte particulière de la joie.

\section{Conclusion}

Que retiendra-t-on de Génération 2016? Le souvenir de musiques hautement poétiques fermement ancrées dans la recherche sonore. Chez Henry, ce sera le souvenir de la gestuelle graphique et l'organicité. Pour Brook, l'espace et l'architecture. De la musique de Schroeder, on gardera les grondements viscéraux de ses instruments, et pour Brook, finalement, la couleur de ses harmonies. Il y a longtemps que la preuve de l'existence de vases communicants entre musiques électroacoustique et instrumentale n'est plus à faire, et Génération 2016 est un exemple parmi tant d'autres que l'intérêt pour le timbre en musique contemporaine instrumentale demeure une pratique actuelle.

Finalement, mentionnons qu'il aurait été impossible d'atteindre un concert d'une telle qualité sans le travail admirable d'un ensemble solidement dirigé. Après tout, ce sont eux qui prennent le risque en «jouant»sur les zones liminales entre l'abstraction de la partition et le concret du son produit. Ainsi, aux compositeurs de Génération 2016, à l'ECM+ et à Véronique Lacroix, nous tirons notre chapeau.
1. Deux publications récentes donnent le détail de ce projet. L'album anniversaire publié en 2014 par l'ECM + retrace l'évolution de Génération et comporte des témoignages de tous les participants: Ensemble contemporain de Montréal, 20 ans de Génération/Ateliers \& Concert: 1994-2014, <www.ecm.qc.ca/ecm. php ? page $=$ precedents\&lang $=f>$ (consulté le 9 décembre 2016). Un article se concentre sur le travail des quatre compositeurs sélectionnés pour Génération 2014 : Symon Henry (2015), "Regards sur les quatre compositeurs lauréats", $1^{\text {re }}$ et $2^{\mathrm{e}}$ parties, Circuit, musiques contemporaines, vol. 25, $\mathrm{n}^{\text {os }} 1$ et 2, p. 86-93 et 77-81.

2. Cette introduction a été écrite avec Bruno de Cat. Nous le remercions pour sa contribution.

3. Citons le Forum du NEM, l'Atelier pour les compositeurs émergents (Emerging Composers Workshop de Soundstreams) ou encore l'IMPULS Academy.

4. Ici, le terme "électroacoustique" référera particulièrement aux pratiques concrète et acousmatique.

5. Voir <https://symonhenry.com> (consulté le g décembre 2016).

6. Georges Boeuf (2008), Entrevue avec Georges Boeuf, $<$ http://georges.boeuf.free.fr/img/presse/interview_gboeuf_ octobreo8.pdf> (consulté le 8 décembre 2016).

7. Michel Chion (1972), " 20 années de musique électro-acoustique, ou une quête d'identité", Musique en jeu, nº 8, p. 21.

8. Voir <www.adamscime.com> (consulté le 9 décembre 2016).

9. Voir ArtWiki [s.d.], François Bayle, <https://goo.gl/zNqo67> (consulté le 9 décembre 2016).

10. Voir <http://sabrinaschroeder.com> (consulté le 9 décembre 2016).

11. Voir <http://taylorbrookmusic.com> (consulté le 9 décembre 2016).

12. Pour comprendre la notion de cent, imaginons un piano qui aurait 98 touches entre les notes do et ré. 